\title{
Elevated methylation of the $R X R A$ promoter region may be responsible for its downregulated expression in the myocardium of patients with TOF
}

\author{
Jing Zhang ${ }^{1,2}$, Xiaojing Ma ${ }^{1,2}$, Huijun Wang ${ }^{1,2}$, Duan Ma ${ }^{2}$ and Guoying Huang ${ }^{1,2}$
}

BACKGROUND: As an important component of retinoid acid signaling pathway, the retinoid $X$ receptor $\alpha$ (RXRA) is considered to play an important role in the pathogenesis of tetralogy of Fallot (TOF).

METHODS: The expression level of RXRA mRNA and the methylation status of the RXRA promoter region in 26 patients with TOF and 6 controls were detected using real-time PCR and bisulfite-specific PCR and cloning-based sequencing, respectively. Dual-luciferase reporter assays, combined with in vitro methylation assay, were performed to determine the transcriptional regulatory activity of unmethylated and methylated CpG regions in the RXRA promoter.

RESULTS: The mRNA expression of RXRA in the right ventricular outflow tract (RVOT) myocardium was significantly decreased in patients with TOF compared with that in the controls. The methylation status of region -1453 to -1000 containing CpG sites 1-23 in the RXRA promoter region was higher in patients with TOF than that in the controls. This region contained several transcription factor sites. In addition, dual-luciferase reporter assays combined with methylation assay in vitro showed that this region had transcriptional regulatory activity, which can be depressed by the methylation of this region.

CONCLUSION: The elevated methylation at RXRA promoter may be responsible for the downregulated mRNA expression in RVOT myocardium of patients with TOF.

C onotruncal defects were thought to be induced by abnormal development of the outflow tract during embryogenesis (1). Tetralogy of Fallot (TOF) is the most common type of conotruncal defects, with an incidence estimated to be 2.5-3.5 per 10,000 live births (2). It is characterized by pulmonary outflow tract obstruction, ventricular septal defects, overriding aortic roots, and right ventricular hypertrophy (3). The etiology of TOF is multifactorial, implying contributions from sequence variations, anomalous gene expression, and epigenetic factors, as well as environmental contributions. Functional mutations of several important cardiac-related transcriptional factor genes and chromosomal abnormality were identified in patients with TOF, supporting a genetic contribution (4-6). In spite of the expanding knowledge of the genetic mechanisms involved in cardiac formation, there remain nearly $80 \%$ of children with congenital heart defects who do not have a known genetic defect. A number of researches have indicated that altered gene expression in signaling pathways regulates heart development and contributes to the development of TOF (7-9). The changed expression of cardiac-related genes may be an essential component contributing to the pathogenesis of TOF. Despite improvements in clarifying the pathogenesis of TOF to some extent, a specific explanation is lacking for the failure of cardiac development in majority of children with TOF.

It has been clarified that adequate retinoid acid (RA) is essential for multiple aspects of cardiogenesis including outflow tract formation. Based on embryos that have had attenuated endogenous RA signaling, it has been demonstrated that components of the RA signaling pathway are necessary for the progression of cardiac morphogenesis (10-15). The RA signaling pathway is primarily mediated via two classes of nuclear receptors: retinoic acid receptors (RARs) and retinoic $\mathrm{X}$ receptors (RXRs). Both the classes have $\alpha, \beta$, and $\gamma$ subclasses (16). As the most important components of RA signaling pathway, the retinoid receptors may play important roles in cardiogenesis including development of the outflow tract.

RXRs have unique characteristics that distinguish them from RARs. First, RXRs mediate the heterodimerization with RARs and are essential for the functional activation of RARs by their ligands. Second, RXRs have the ability to form homodimers with themselves or heterodimers with other nucleic receptors such as thyroid hormone receptors, peroxisome proliferator-activated receptors, vitamin $\mathrm{D}$ receptor, and liver X receptor (17-19). Thus, RXRs may be a constituent in multiple hormonal signaling pathways and mediate expression of a large variety of hormone-responsive genes. In addition, specific functions of the different retinoid receptors during heart embryogenesis have been identified using genetic approaches. Single mutations of $R A R$ in mice had no effect on heart development (20-22), whereas RXRA (retinoid X receptor $\alpha) / R A R$ double-mutant mice exhibited abnormal heart 
development including TGA (23). RXRA mutant or knockout mice displayed a wide range of outflow tract abnormalities (24-26), whereas $\mathrm{RXR} \beta^{-/-}$null mice, $\mathrm{RXR} \gamma^{--}$null mice, $\mathrm{RXR} \beta^{-1-} / \mathrm{RXR} \gamma^{--}$double-null mutant mice, and compound $\mathrm{RXR}^{+/-} \mathrm{RXR} \beta^{-1-} / \mathrm{RXR} \gamma^{-1}$ mutant mice were viable, displaying no obvious congenital heart abnormalities (27). These studies suggest that RXRA is the functionally most important RXR isoform implicated in cardiac outflow tract development.

However, although animal studies have suggested a potentially important role for RXRA in the pathogenesis of conotruncal defects, there has been no evidence showing the same role for RXRA in patients with TOF. Moreover, it remains unclear how RXRAs take part in the pathogenesis of TOF. Thus, this study was designed to illustrate the relationship between patients with TOF and RXRA and the role of RXRA in the pathogenesis of TOF by preliminarily investigating the expression pattern of RXRA mRNA in the right ventricular outflow tract (RVOT) myocardium of patients with TOF and subsequently detecting the methylation status of RXRA gene promoter.

\section{RESULTS}

\section{Expression of RXRA mRNA}

Real-time PCR analysis revealed that the mRNA expression of $R X R A$ in the RVOT myocardium was significantly decreased in patients with TOF compared with that in the controls (mean \pm SD: $0.218 \pm 0.098$ and $0.816 \pm 0.210$, respectively; $P=0.0002$; Figure 1). The relative fold change in the transcript expression of RXRA was significantly downregulated by nearly 3.7 -fold in patients with TOF compared with the controls. The mRNA expression levels of the 6 individuals in the control cohort were distributed over a relatively wide range from 0.525 to 1.058 , whereas the mRNA expression levels of the 26 patients with TOF were centralized within a small range.

\section{Methylation Status of $\mathrm{CpG}$ Sites in the RXRA Promoter}

The methylation status of region -1453 to 374 with respect to the transcription start site (TSS) containing CpG sites 1-97 in

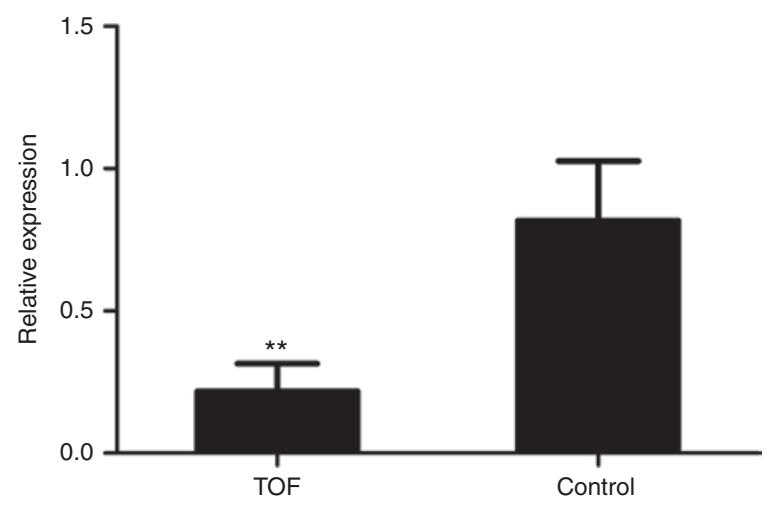

Figure 1. RXRA mRNA expression in human right ventricular outflow tract (RVOT) tissue. Relative expression of RXRA mRNA was quantified by realtime PCR in RNAs obtained from RVOT tissue of 26 tetralogy of Fallot (TOF) children and 6 controls. Data are presented as mean \pm SD of individual data of either patients with TOF or controls. ${ }^{* *} P<0.01$, Mann-Whitney test. RXRA, retinoid $X$ receptor $\alpha$. the RXRA promoter region was measured by bisulfite-specific PCR (BSP) and cloning-based sequencing. When compared with the controls, the patients with TOF had a higher methylation percentage nearly at all sites, but only $\mathrm{CpG}$ sites 2 (mean \pm SD: $88.5 \pm 8.8$ and $76.7 \pm 10.3$, respectively; $P=0.0159), 13$ (mean \pm SD: $27.3 \pm 12.2$ and $5.0 \pm 5.5$, respectively; $P=0.0007$ ), and 20-23 (mean \pm SD: $5.8 \pm 6.0$ and $0.8 \pm 1.3$, respectively; $P=0.0123$ ) had statistically significant differences (Figure 2). As per analysis, screening of all $97 \mathrm{CpG}$ sites of the RXRA promoter region revealed that the overall methylation percentage (CpG sites 1-97) was a little higher in the patients with TOF compared with that in the controls (mean \pm SD: $5.5 \pm 1.3$ and $3.4 \pm 0.8$, respectively; $P=0.0028$ ). However, we found that the $\mathrm{CpG}$ region containing $\mathrm{CpG}$ sites 24-97 was hypomethylated, where the methylation percentage was low $(<5 \%)$ in both patients with TOF and the controls at almost every CpG site. The overall methylation status within $\mathrm{CpG}$ region containing CpG sites 1-23 was statistically significantly higher in patients with TOF compared with that in the controls (mean \pm SD: $17.0 \pm 2.7$ and $13.0 \pm 3.4$, respectively; $P=0.0156$, Figures 3 and 4). We searched online (http://www.cbrc.jp/research/db/ TFSEARCH.html) and found that the region from -1453 to -1000 containing CpG sites 1-23 contained several transcription factor sites (Figure 5).

\section{Methylation Status of Inserted CpG Sites in PGL3-RXRA-Promoter}

BSP and cloning-based sequencing were used to determine the methylation status of the unmethylated pGL3-RXRAPromoter and methylated pGL3-RXRA-Promoter after CpG methyltransferase (M.Sss I) treatment in vitro. Before M.Sss I treatment, the overall methylation status of the inserted 23 CpG sites was 9.6\%. After M.Sss I treatment, $94.8 \%$ of the $\mathrm{CpG}$ sites were methylated (Figure 6).

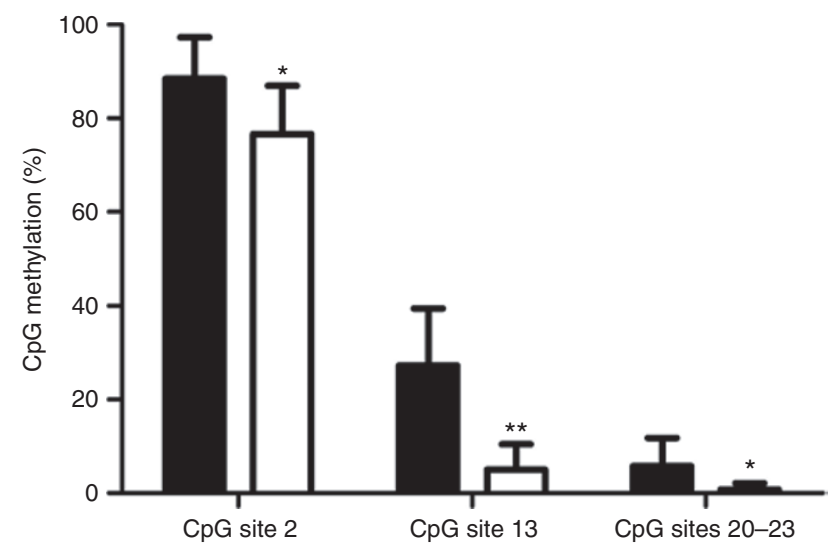

Figure 2. Methylation status at CpG sites 2, 13, and 20-23 in RXRA promoter. DNA methylation analysis of RXRA for CpG sites 2, 13, and 20-23 was performed, and the percentage of $\mathrm{CpG}$ sites methylated to the total number of $\mathrm{CpG}$ sites assessed for the sites was examined. $x$-Axis represents the number of $\mathrm{CpGs}$ sites, whereas $y$-axis represents the percentage of $\mathrm{CpG}$ methylation. The black fill represents data of tetralogy of Fallot (TOF) group, whereas the white fill represents data of control group. Significant differences of control vs. TOF groups are presented. ${ }^{*} P<0.05$, ${ }^{* *} P<0.01$, Mann-Whitney test. RXRA, retinoid X receptor $\alpha$. 


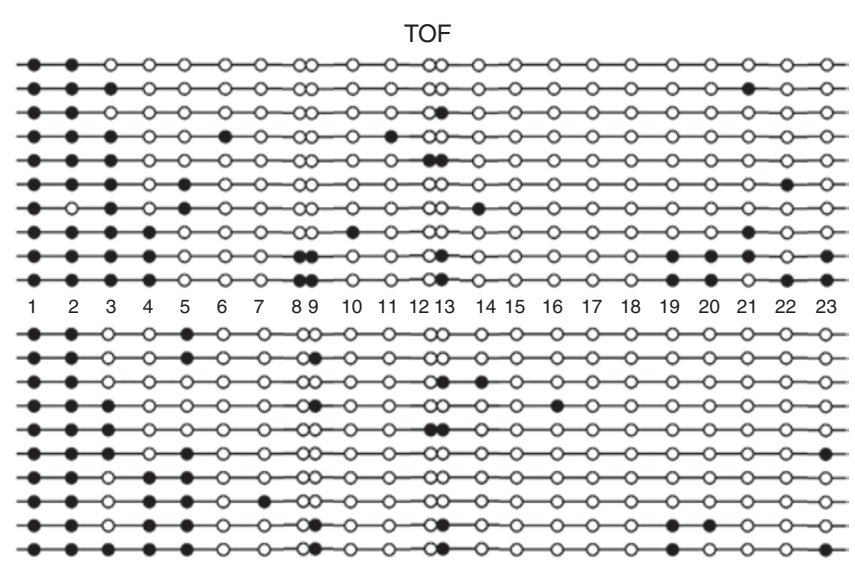

Control

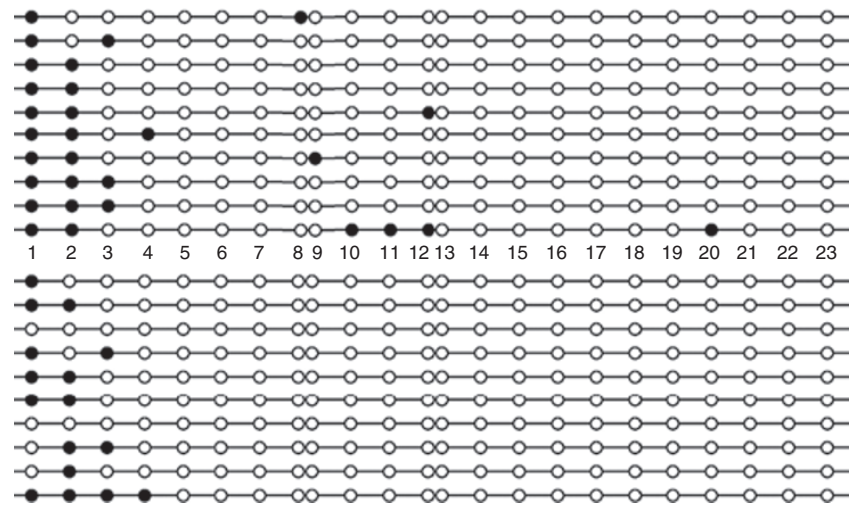

Figure 3. Bisulfite-specific PCR (BSP) and cloning-based sequencing results of CpG sites 1-23 in RXRA promoter. BSP and cloning-based sequencing were performed to analyze 10 clones from 26 individual DNA samples from the tetralogy of Fallot (TOF) group and 6 individual DNA samples from the control group. Methylation patterns of CpG sites 1-23 from two cases in both the groups are represented by circles. Each line represents a single clone. All unmethylated $\mathrm{CpGs}$ sites are represented as white circles, whereas methylated $\mathrm{CpGs}$ sites are represented as black circles. RXRA, retinoid X receptor $\alpha$.

\section{Transcriptional Regulatory Activity of -1453 to -1000 Region in the RXRA Promoter}

The normalized luciferase activity of pGL3-RXRA-Promoter was significantly higher than that of pGL3-Promoter (Figure 7a; $P<0.0001$ ). The normalized luciferase activity of methylated pGL3-RXRA-Promoter (mpGL3-RXRA-Promoter) decreased by nearly threefold (Figure $7 \mathbf{b} ; P<0.0001$ ) compared with that of pGL3-RXRA-Promoter.

\section{DISCUSSION}

Animal studies suggested a potentially important role for RXRA in the pathogenesis of TOF. In our previous study, we performed direct sequencing analysis of $R X R A$ in 213 patients with TOF but found no functional variations in the coding region. Interestingly, in this study, downregulation of $R X R A$ mRNA was found in patients with TOF compared with controls, indicating that developmental deficiencies resulting in TOF are associated with distinct changes in gene expression of RXRA. However, little is known about the transcriptional regulation of $R X R A$ gene expression in patients with TOF.

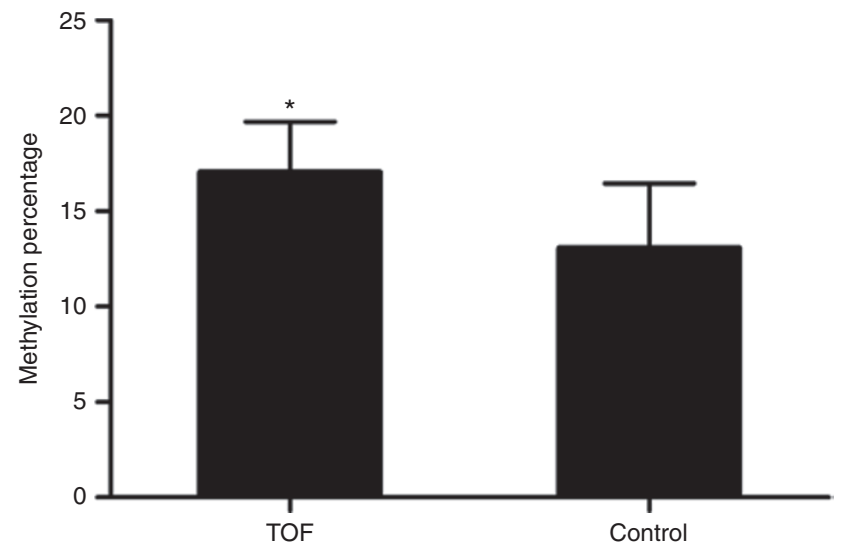

Figure 4. Overall methylation status of CpG sites 1-23 in $R X R A$ promoter. DNA methylation level at all 23 CpG sites from each sample was calculated as the average of the methylation level of each CpG site. Significant difference between control vs. tetralogy of Fallot (TOF) groups is presented. ${ }^{*} P<0.05$, Mann-Whitney test. RXRA, retinoid X receptor $\alpha$.

Because a better understanding of the mechanisms responsible for RXRA gene expression will help decipher the pathogenesis of TOF and provide new opportunities for therapy, we further focused on elucidating the molecular basis for the transcriptional regulation of RXRA gene expression.

At the transcriptional level, the regulation of gene expression is mediated by cis-acting DNA sequences and their associated trans-acting factors. These genomic DNA sequence elements act as binding sites for the transcription machinery such as transcription factors (28). Therefore, sequence variations in these binding sites may interrupt the binding activity of transcription machinery, leading to abnormal transcriptional expression. In our previous study, the promoter regions of $R X R A$ gene were genetically analyzed in 213 patients with TOF and 500 healthy controls. One novel heterozygous mutation, A-1191G (according to the TSS), was found in one of the patients with TOF but in none of the controls. The A at position -1191 follows C, so the mutation A-1191G provided additional candidate cytosines $(\mathrm{CpG})$ for methylation that may influence the level of gene expression (see Supplementary Data online). To identify the function of this mutation in the pathogenesis of TOF, further research is needed.

Other than DNA sequence variations in regulatory elements, chromatin-level gene regulation, which is an epigenetic mechanism that controls gene expression without changes in the DNA sequence, was found to play essential roles in heart development (29-32). DNA methylation is the best characterized epigenetic mechanism, which involves the covalent addition of a methyl group to the cytosine base within the context of $\mathrm{CpG}$ dinucleotides. CpG-rich islands, short stretches of DNA with a relatively high frequency of $\mathrm{CpG}$ sites, are often found in promoters of mammalian genes. The increased level of methylation at these sites is believed to repress the transcriptional expression of corresponding genes (33). A study using mouse model for colon cancer showed that the reduction in the RXRA levels correlated with an increase in the methylation status of the promoter region of the $R X R A$ gene 


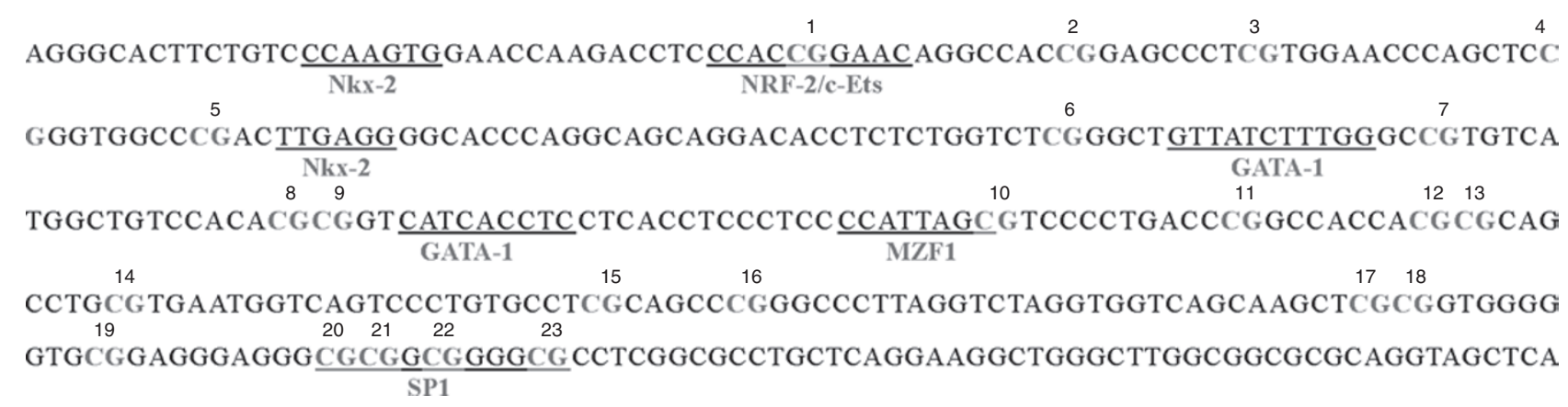

Figure 5. The putative transcription factor binding sites of -1453 to -1000 region containing $\mathrm{CpG}$ sites $1-23$ in RXRA promoter region. The predicted transcription factor binding sites in fragment of $R X R A$ promoter region from -1453 to -1000 by using the TFSEARCH program. RXRA, retinoid $X$ receptor $\alpha$.

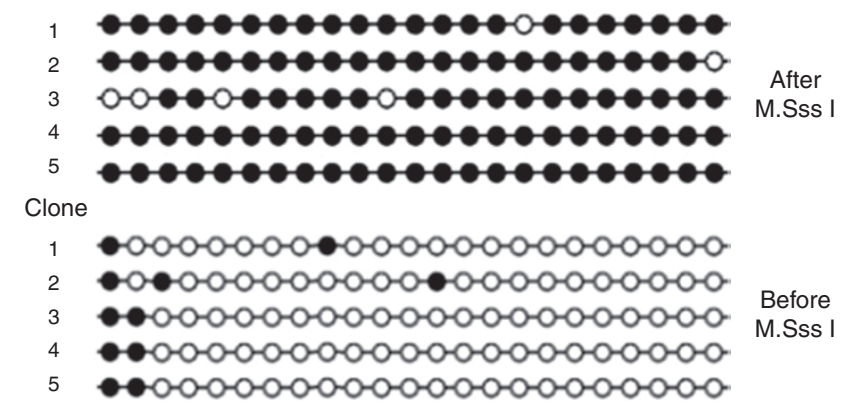

Figure 6. The bisulfite-specific PCR (BSP) and cloning-based sequencing of unmethylated and methylated PGL3-RXRA-Promoter. BSP and cloningbased sequencing were used to determine the methylation status of the unmethylated pGL3-RXRA-Promoter and methylated pGL3-RXRA-Promoter after M.Sss I treatment in vitro. For each plasmid, the methylation of $\mathrm{CpG}$ sites 1-23 is shown for five clones. White and black circles indicate unmethylated and methylated $\mathrm{CpGs}$, respectively. The results show that after M.Sss I treatment, $94.8 \%$ of the CpG sites were methylated. RXRA, retinoid $X$ receptor $\alpha$.

(34). Similarly, in this study, we found that the overall methylation status of RXRA promoter region was statistically significantly higher in patients with TOF compared with that in the controls. These data suggested a potential role for the methylation of $R X R A$ promoter region in regulating the expression of RXRA mRNA.

In this study, we found that the overall methylation status of the region between positions -1453 and -1000 , which contains CpG sites 1-23, was statistically significantly higher in patients with TOF compared with that in the controls. In a previous study, Li et al. (35) identified the promoter of human RXRA gene and found a regulatory region between positions -1465 and -1082 , which showed enhanced transcriptional regulatory activity. In this study, we found that the normalized luciferase activity of pGL3-RXRA-Promoter was significantly higher than that of pGL3-Promoter for 293T cell line, which indicated that the region between positions -1453 and -1000 had enhanced transcriptional regulatory activity. By using TFSEARCH software v.1.3 (Parallel Application TRC Laboratory, RWCP, Japan), a series of putative transcription factor binding sites was found spanning from -1453 to -1000 in $R X R A$ promoter. Taken together, the findings suggested that the region between positions -1453 and -1000 was an important regulatory region in $R X R A$ promoter and that it had enhanced transcriptional activity. The increased methylation level of this region may take an important part in regulating the expression of RXRA mRNA.

To investigate whether the observed downregulation of mRNA expression of RXRA may be due to an increased methylation grade of the region between positions -1453 and -1000 containing CpG sites 1-23 in the RXRA promoter region, dual-luciferase reporter assays combined with in vitro methylation assay were performed. After in vitro methylation, the transcriptional activity of pGL3-RXRA-Promoter was significantly decreased, indicating that increased methylation level of region -1453 to -1000 in $R X R A$ promoter can depress its transcriptional regulatory activity.

Methylation of the promoter region is associated with compacted chromatin structure, which depresses the affiliated gene expression by directly preventing the access of transcription factors or indirectly recruiting a transcriptional corepressor (36-39). Further researches are necessary to identify how the increased methylation level of the region between positions -1453 and -1000 in RXRA promoter depresses the transcriptional expression of RXRA mRNA. What is more, in this study, we mainly focused on the region-specific methylation status of the RXRA promoter. Methylation modification at special CpG residues should be further analyzed to elucidate the potentially affected affinity for transcription factors of neighboring cis elements.

\section{Conclusion}

The majority of congenital heart defects are thought to result from the interaction between multiple genetic, epigenetic, environmental, and lifestyle factors.

Understanding the factors and mechanisms that regulate gene expression in the heart development is essential for the detection, treatment, and prevention of congenital heart diseases. To our knowledge, this is the first report that shows that there is downregulation of RXRA mRNA expression and elevated promoter methylation status in the RVOT myocardium of patients with TOF. The elevated methylation at RXRA promoter may be responsible for the downregulated gene expression in RVOT myocardium of patients with TOF. These findings may support a possible role for RXRA in the pathogenesis and development of TOF. 
a

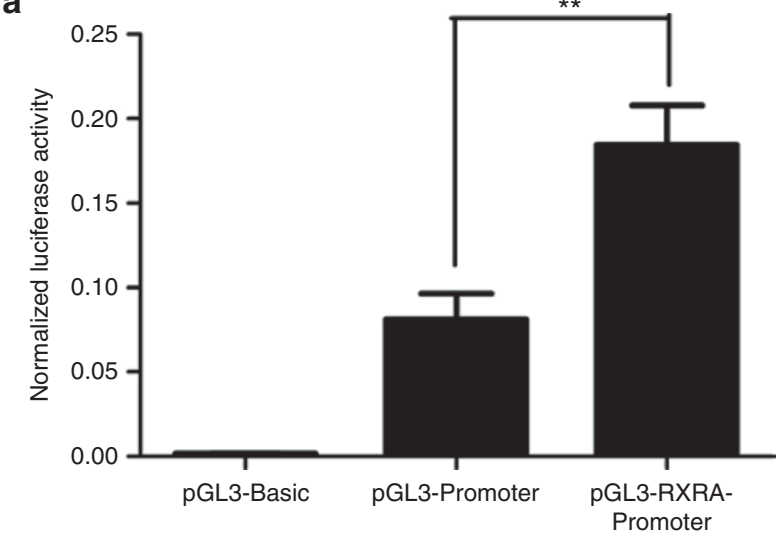

b

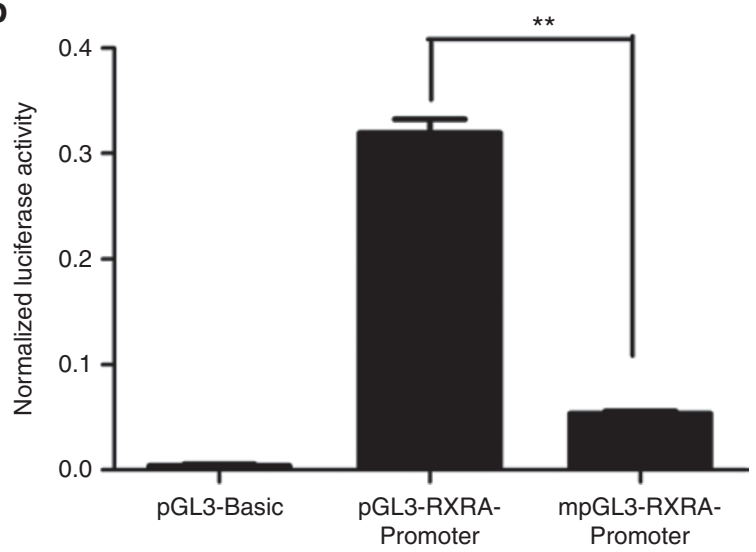

Figure 7. Enhanced transcriptional regulatory activity of pGL3-RXRA-Promoter and decreased transcriptional regulatory activity of mpGL3-RXRA-Promoter. (a) The -1453 to -1000 region containing CpG sites 1-23 in RXRA promoter is important for RXRA promoter activity. The effect of the -1453 to -1000 region on the regulation of $R X R A$ promoter transcriptional activity is shown. pGL3-Basic plasmid was used as the negative control. Values of luciferase activities for each plasmid were normalized for transfection efficiency by cotransfection with pRL-TK plasmid and are shown as mean \pm SD represented for three independent experiments, each of which was performed using triplicate samples. ${ }^{* *} P<0.01$, Mann-Whitney test. (b) Effect of in vitro methylation on the transcriptional regulatory activity of the -1453 to -1000 region containing CpG sites 1-23 in RXRA promoter. The pGL3-RXRA-Promoter plasmid was methylated in vitro by M.Sssl methylase. Methylated pGL3-RXRA-Promoter plasmid (mpGL3-RXRA-Promoter) and unmethylated pGL3-RXRA-Promoter plasmid were then transiently transfected into HEK293T cells and assayed for luciferase activity (as mentioned in a). The results are shown as mean \pm SD represented for three independent experiments, each of which was performed using triplicate samples. ${ }^{* *} P<0.01$, Mann-Whitney test.

Table 1. Primers for real-time PCR

\begin{tabular}{llc}
\hline Primers & \multicolumn{1}{c}{ Sequences $\left(5^{\prime}-3^{\prime}\right)$} & $\begin{array}{c}\text { Product } \\
\text { size }(\mathrm{bp})\end{array}$ \\
\hline RXRA-RT-F & AGATGGACAAGACGGAGCTG & 120 \\
RXRA-RT-R & CCAAGGACGCATAGACCTTC & \\
GAPDH-RT-F & CACCCACTCCTCCACCTTTG & 108 \\
GAPDH-RT-R & ACCACCCTGTTGCTGTAGCC & \\
\hline
\end{tabular}

F, forward; GAPDH, glyceraldehyde-3-phosphate dehydrogenase; R, reverse; RXRA, retinoid $X$ receptor $\alpha$

\section{METHODS}

\section{Subjects and Tissue Collection}

Patients with TOF were recruited from the Children's Hospital of Fudan University, Shanghai, China. These patients were diagnosed using an echocardiogram, and the diagnoses were confirmed by surgery. RVOT tissue samples were collected from 26 patients with TOF undergoing surgical reconstruction, including 18 (69.2\%) male and 8 (30.8\%) female patients of age 5-48 mo (mean \pm SD: $15.8 \pm 13.0 \mathrm{mo}$ ). Human samples were collected from consenting individuals according to the protocols approved by the Ethics Committee of Children's Hospital of Fudan University.

The control subjects were recruited from autopsy specimens at the Forensic Medicine Department of the Fudan University, Shanghai, China. Normal RVOT tissue samples were obtained from six control subjects who had died as a result of traffic accidents, including four $(66.7 \%)$ men and two $(33.3 \%)$ women of age 6-48 mo (mean \pm SD: $21.0 \pm 17.3 \mathrm{mo}$ ). The next-of-kin of the deceased signed the autopsy and sample collection form. The research program was permitted by the Shanghai Medical College of Fudan University.

The tissues were saved in RNAlater Solution (Ambion, Austin, TX) immediately after surgical removal or autopsy and stored at $-80^{\circ} \mathrm{C}$ until use. Detailed information is shown in Supplementary Table S1 online.

\section{Cell Line}

HEK293T cells (human embryonic kidney) were maintained in our laboratory and grown in Dulbecco's modified eagle medium (Gibco BRL, Langley, OK) supplemented with $10 \%(\mathrm{v} / \mathrm{v})$ fetal bovine serum (Gibco BRL). All the cells were cultured at $37^{\circ} \mathrm{C}$ in $5 \% \mathrm{CO}_{2}$.
Table 2. Primers for BSP

\begin{tabular}{|c|c|c|c|}
\hline Primers & & Sequences $\left(5^{\prime}-3^{\prime}\right)$ & $\begin{array}{l}\text { Product } \\
\text { size (bp) }\end{array}$ \\
\hline \multirow[t]{4}{*}{ Fragment 1} & Outside F & GTTTTGGGTTTTGGTTGTG & 651 \\
\hline & Outside R & TAAAACCCCTCTTCAAAAACAC & \\
\hline & Inner F & AGGGTATTTTTTGTTTTAAGTGG & 285 \\
\hline & Inner R & ACCACCTAAACCTAAAAACC & \\
\hline
\end{tabular}

Fragment 2 OutsideF GTTTTGGGTTTTGGTTGTG

Outside $R$ TAAAACCCCTCTTCAAAAACAC

Inner F TGAATGGTTAGTTTTTGTGTT

Inner $\mathrm{R} \quad$ AATTCTCTACCTAACTTTTAAATTCA

Fragment 3 Outside F GGGTTTTTAGGTTTAGGTGGTT

Outside R CAACRACAAAAAACAAACTCC

Inner F GGGGTAGGAGTGTTTTTTTT

Inner R CTACCCCCACRAAAAAACC

Fragment 4 Outside F TGTTTAGGAAGGTTGGGTTTG

Outside R AACRAAAACAAAACTCCCTCC

Inner F TTTGGTGTTTTTGAAGAGGG

Inner R ACCAAACAAACRCAAAACC

Fragment 5 Outside F TGTTTAGGAAGGTTGGGTTTG

Outside R AACRAAAACAAAACTCCCTCC

Inner F TTTTTTGTYGTTGGGGTATAT

Inner R AAAACAAAACTCCCTCCC

Fragment 6 Outside F GGTTTTGCGTTTGTTTGGTT

Outside R ACGAAAAACCGACGACTACG

Inner F GGGAGGGAGTTTTGTTTT

Inner R AACRCTAACTCRACRCAA

BSP, bisulfite-specific PCR; F, forward; R, reverse; RXRA, retinoid X receptor $\alpha$. 
Table 3. Primers for amplification of -1453 to -1000 region of $R X R A$

\begin{tabular}{lcc}
\hline Primers & Sequences $\left(5^{\prime}-3^{\prime}\right)$ & $\begin{array}{c}\text { Product } \\
\text { size }(\mathrm{bp})\end{array}$ \\
\hline RXRA-Kpnl-F & GATC GGTACC AGGGCACTTCTGTCCCAAGTG & 362 \\
RXRA-Xhol-R & GATC CGGAGC CGCGGACGAGTCCTTGAGCTC & \\
\hline
\end{tabular}

$F$, forward; R, reverse; RXRA, retinoid X receptor $\alpha$.

Table 4. Primers for BSP of pGL3-RXRA-Promoter

\begin{tabular}{llc}
\hline Primers & \multicolumn{1}{c}{ Sequences $\left(5^{\prime}-3^{\prime}\right)$} & $\begin{array}{c}\text { Product } \\
\text { size }(\mathrm{bp})\end{array}$ \\
\hline Forward & YGATAGGTATTAGGGTATTTTTG & 369 \\
Reverse & ATCTCRAATTCCTAAACAAAC & \\
\hline
\end{tabular}

BSP, bisulfite-specific PCR; RXRA, retinoid X receptor $\alpha$.

\section{RNA/DNA Isolation and Treatments}

Total RNA was isolated from RVOT tissue using Trizol Reagent (Invitrogen, Carlsbad, CA). Agarose gel electrophoresis (1.5\%) was used to check for RNA degradation and DNA contamination. For each sample, $500 \mathrm{ng}$ of total RNA was used for cDNA synthesis with PrimeScript RT reagent Kit (Takara Biotechnology, Dalian, China) in a $10-\mu \mathrm{l}$ reaction mix in accordance with the manufacturer's instructions. The final cDNA product was diluted 10 -fold and subsequently used as templates for real-time PCR.

Genomic DNA was extracted from $30 \mathrm{mg}$ (weight) of RVOT tissue by using QIAamp DNA Micro Kit (Qiagen, Hilden, Germany) according to the manufacturer's instructions. For each sample, $1 \mu \mathrm{g}$ of gnomic DNA obtained from RVOT tissue was modified using EZ DNA Methylation-Gold Kit (Zymo Research, Irvine, CA) following the manufacturer's instructions. Sodium bisulfite-treated genomic DNA was used as templates for BSP.

The quality and integrity of the RNA/DNA were determined by the A260/280 ratio using ND-1000 Nanodrop (NanodropTech, Wilmington, DE).

\section{Real-Time PCR}

The cDNA sequencefor RXRA (GeneBankaccession no.NM_002957.4) was obtained from the National Center for Biotechnology Information's GeneBank, and the primers (Table 1) for real-time PCR were designed using online Primer3 software (v.0.4.0) (Whitehead Institute for Biomedical Research, Cambridge, MA). Amplification reactions (detailed information is shown in Supplementary Table S2 online) were carried out using the SYBR Premix Ex Taq Kit (Takara Biotechnology) and were performed in triplicates in 384-well-plate format using an Applied Biosystems 7900HT Fast Real-time PCR System (Applied Biosystems, Foster City, CA).

Relative quantification of gene expression was calculated using the $2^{-\Delta \Delta \mathrm{Ct}}$ method. Glyceraldehyde-3-phosphate dehydrogenase expression levels were used for normalization of gene expression values.

\section{CpG Island Analysis}

The promoter region of RXRA (2,000 bp upstream of the TSS) was analyzed for the presence of $\mathrm{CpG}$ islands using Methyl Primer Express v1.0 software (Applied Biosystems). The criteria for CpG islands are (i) a region of DNA greater than $200 \mathrm{bp}$, (ii) GC content above 0.5 , and (iii) observed/expected CpG ratio above 0.6. By these criteria, a $\mathrm{CpG}$ island spanning from -1453 to -38 with respect to the TSS was predicted.

\section{BSP and Cloning-Based Sequencing}

The methylation statuses were measured using BSP and cloningbased sequencing, which are considered to be the gold standards for methylation evaluation. The RXRA CpG island was divided into six fragments (F1-F6; see Supplementary Figure S1 online) amplified by the specific primer sets (Table 2) that were designed using Methyl Primer Express v1.0 software. To amplify a specific product, a nested PCR analysis was performed. Detailed information is shown in Supplementary Table S3 online. Following nested PCR amplification, $2 \mu \mathrm{l}$ of each PCR product was visualized on a standard $2.0 \%$ agarose gel and the remaining $48 \mu \mathrm{l}$ was purified using the AxyPrep DNA Gel Extraction Kit (Axygen, Union City, CA). Purified PCR products were cloned into a pTA2 vector (TOYOBO, Osaka, Japan) and were then transformed into DH5 $\alpha$ competent cells (TIANGEN, Beijing, China). Blue/white and ampicillin screening were performed after $12 \mathrm{~h}$ of incubation at $37^{\circ} \mathrm{C}$. Plasmids extracted and purified from 10 white colonies for each sample were sequenced in both directions using M13 forward or reverse primer by Jie Li Biology (Shanghai, China).

\section{Sequencing Data Analysis}

The sequencing data obtained from BSP and cloning-based sequencing were analyzed using BIQ Analyzer software (Saarbrücken, Germany). The methylation percentage of each $\mathrm{CpG}$ site in a given sample was calculated as the number of the methylated $\mathrm{CpG}$ sites divided by the total observed sequenced clone numbers. Overall methylation status of a specific region in a given sample was the average of the methylation status of each CpG site in the DNA region.

As a result of the unsuccessful amplification of fragment 6, the methylation status of $97 \mathrm{CpG}$ sites (from -1453 to -374 , according to the TSS) at the promoter region of RXRA gene was analyzed.

\section{Plasmid Constructs and In Vitro Methylation}

The $5^{\prime}$ region ( -1453 to -1000 relative to the TSS) of human $R X R A$ was amplified using high-fidelity LA Taq PCR (Takara Biotechnology) from human genomic DNA isolated from the tissue of healthy controls and inserted into the pGL3-Promoter vector (Promega, Madison, WI) to make pGL3-RXRA-Promoter plasmid (see Supplementary Figure S2 online). The primers for PCR amplification are listed in Table 3. The validity of DNA sequence inserted into pGL3-Promoter was confirmed by direct sequencing.

The pGL3-RXRA-Promoter was methylated by incubation with M.SssI (New England BioLabs, Beijing, China) for $3 \mathrm{~h}$ at $37^{\circ} \mathrm{C}$ in the presence of $160 \mu \mathrm{mol} / \mathrm{l} S$-adenosylmethionine. The methylation status was verified by BSP and cloning-based sequencing using the primers listed in Table 4.

\section{Transfections and Luciferase Assays}

For transient transfections, HEK293T cells were plated in 24-well plates $12-14 \mathrm{~h}$ before transfection at a density of $2 \times 10^{5}$ cells/well. HEK293T cells were transfected with $400 \mathrm{ng}$ of pGL3-Basic, pGL3-Promoter, pGL3-RXRA-Promoter (unmethylated), and mpGL3-RXRA-Promoter (methylated), respectively. The pGL3-Basic vector containing no promoter sequences served as the negative control. The pRL-TK plasmid (Promega) containing the renilla luciferase gene driven by the herpes simplex virus thymidine kinase promoter was cotransfected with the plasmids mentioned previously to normalize the luciferase activity. All transient transfections were done with Lipofectamine 2000 transfection reagent (Invitrogen) according to the manufacturer's protocol. The preparation of cell lysates was done using the Dual-Luciferase Reporter Assay System (Promega), and the luciferase activity was measured using Luminoskan TL Plus (Thermo Labsystems, Oy, Helsinki, Finland).

Firefly luciferase activity of individual transfections was normalized against renilla luciferase activity. Each value presented is the average of triplicate samples and is representative of multiple independent experiments.

\section{Statistical Analysis}

Statistical analysis was performed using GraphPad prism software v5 (Graphpad software, San Diego CA). The differences of RXRA expression and methylation percentages between TOF and controls were measured using the Mann-Whitney test. For all analyses, a nominal $P$ value of less than 0.05 was considered statistically significant.

\section{SUPPLEMENTARY MATERIAL}

Supplementary material is linked to the online version of the paper at http:// www.nature.com/pr

\section{STATEMENT OF FINANCIAL SUPPORT}

This work was supported by grants from the National Natural Sciences Foundation of China (30930096), the National Basic Research Program of China 
(2010CB529504), and Shanghai Municipal Commission of Science and Technology research projects (11JC1401400).

Disclosure: The authors have no relevant financial disclosures or other potential conflicts of interest to report.

\section{REFERENCES}

1. Johnson TR. Conotruncal cardiac defects: a clinical imaging perspective. Pediatr Cardiol 2010;31:430-7.

2. Ferencz C, Rubin JD, McCarter RJ, et al. Congenital heart disease: prevalence at live birth. The Baltimore-Washington Infant Study. Am J Epidemiol 1985;121:31-6.

3. Ho S, McCarthy KP, Josen M, et al. Anatomic-echocardiographic correlates: an introduction to normal and congenitally malformed hearts. Heart 2001;86:Suppl 2:13-11.

4. Gelb BD. Genetic basis of congenital heart disease. Curr Opin Cardiol 2004;19:110-5.

5. Liu C, Shen A, Li X, Jiao W, Zhang X, Li Z. T-box transcription factor TBX20 mutations in Chinese patients with congenital heart disease. Eur J Med Genet 2008;51:580-7.

6. Maitra M, Koenig SN, Srivastava D, Garg V. Identification of GATA6 sequence variants in patients with congenital heart defects. Pediatr Res 2010;68:281-5.

7. Kaynak B, von Heydebreck A, Mebus S, et al. Genome-wide array analysis of normal and malformed human hearts. Circulation 2003;107:2467-74.

8. Sharma HS, Peters TH, Moorhouse MJ, van der Spek PJ, Bogers AJ. DNA microarray analysis for human congenital heart disease. Cell Biochem Biophys 2006;44:1-9.

9. Bittel DC, Butler MG, Kibiryeva N, et al. Gene expression in cardiac tissues from infants with idiopathic conotruncal defects. BMC Med Genomics 2011;4:1.

10. Taylor IM, Wiley MJ, Agur A. Retinoic acid-induced heart malformations in the hamster. Teratology 1980;21:193-7.

11. Lammer EJ, Chen DT, Hoar RM, et al. Retinoic acid embryopathy. N Engl J Med 1985;313:837-41.

12. Ratajska A, Zlotorowicz R, Blazejczyk M, Wasiutyñski A. Coronary artery embryogenesis in cardiac defects induced by retinoic acid in mice. Birth Defects Res Part A Clin Mol Teratol 2005;73:966-79.

13. Keegan BR, Feldman JL, Begemann G, Ingham PW, Yelon D. Retinoic acid signaling restricts the cardiac progenitor pool. Science 2005;307:247-9.

14. Collop AH, Broomfield JA, Chandraratna RA, et al. Retinoic acid signaling is essential for formation of the heart tube in Xenopus. Dev Biol 2006;291:96-109.

15. Cipollone D, Amati F, Carsetti R, et al. A multiple retinoic acid antagonist induces conotruncal anomalies, including transposition of the great arteries, in mice. Cardiovasc Pathol 2006;15:194-202.

16. Chambon P. A decade of molecular biology of retinoic acid receptors. FASEB J 1996;10:940-54.

17. Lotan R, Clifford JL. Nuclear receptors for retinoids: mediators of retinoid effects on normal and malignant cells. Biomed Pharmacother 1991;45:14556.

18. Willy PJ, Umesono K, Ong ES, Evans RM, Heyman RA, Mangelsdorf DJ. LXR, a nuclear receptor that defines a distinct retinoid response pathway. Genes Dev 1995;9:1033-45.

19. Perlmann T, Evans RM. Nuclear receptors in Sicily: all in the famiglia. Cell 1997;90:391-7.
20. Li E, Sucov HM, Lee KF, Evans RM, Jaenisch R. Normal development and growth of mice carrying a targeted disruption of the alpha 1 retinoic acid receptor gene. Proc Natl Acad Sci USA 1993;90:1590-4.

21. Lohnes D, Kastner P, Dierich A, Mark M, LeMeur M, Chambon P. Function of retinoic acid receptor gamma in the mouse. Cell 1993;73:643-58.

22. Luo J, Pasceri P, Conlon RA, Rossant J, Giguère V. Mice lacking all isoforms of retinoic acid receptor beta develop normally and are susceptible to the teratogenic effects of retinoic acid. Mech Dev 1995;53:61-71.

23. Kastner P, Grondona JM, Mark M, et al. Genetic analysis of RXR alpha developmental function: convergence of RXR and RAR signaling pathways in heart and eye morphogenesis. Cell 1994;78:987-1003.

24. Sucov HM, Dyson E, Gumeringer CL, Price J, Chien KR, Evans RM. RXR alpha mutant mice establish a genetic basis for vitamin A signaling in heart morphogenesis. Genes Dev 1994;8:1007-18.

25. Gruber PJ, Kubalak SW, Pexieder T, Sucov HM, Evans RM, Chien KR. RXR alpha deficiency confers genetic susceptibility for aortic sac, conotruncal, atrioventricular cushion, and ventricular muscle defects in mice. J Clin Invest 1996;98:1332-43

26. Kubalak SW, Hutson DR, Scott KK, Shannon RA. Elevated transforming growth factor beta2 enhances apoptosis and contributes to abnormal outflow tract and aortic sac development in retinoic $\mathrm{X}$ receptor alpha knockout embryos. Development 2002;129:733-46.

27. Krezel W, Dupé V, Mark M, Dierich A, Kastner P, Chambon P. RXR gamma null mice are apparently normal and compound RXR alpha $+/-/$ RXR beta -/-/RXR gamma -/- mutant mice are viable. Proc Natl Acad Sci USA 1996;93:9010-4.

28. Ohler U, Wassarman DA. Promoting developmental transcription. Development 2010;137:15-26.

29. Srivastava D. Making or breaking the heart: from lineage determination to morphogenesis. Cell 2006;126:1037-48.

30. Han P, Hang CT, Yang J, Chang CP. Chromatin remodeling in cardiovascular development and physiology. Circ Res 2011;108:378-96.

31. van Weerd JH, Koshiba-Takeuchi K, Kwon C, Takeuchi JK. Epigenetic factors and cardiac development. Cardiovasc Res 2011;91:203-11.

32. Ohtani K, Dimmeler S. Epigenetic regulation of cardiovascular differentiation. Cardiovasc Res 2011;90:404-12.

33. Bird AP. CpG-rich islands and the function of DNA methylation. Nature 1986;321:209-13.

34. Volate SR, Muga SJ, Issa AY, Nitcheva D, Smith T, Wargovich MJ. Epigenetic modulation of the retinoid $\mathrm{X}$ receptor alpha by green tea in the azoxymethane-Apc Min/+ mouse model of intestinal cancer. Mol Carcinog 2009;48:920-33.

35. Li G, Yin W, Chamberlain R, et al. Identification and characterization of the human retinoid X receptor alpha gene promoter. Gene 2006;372:118-27.

36. Comb M, Goodman HM. CpG methylation inhibits proenkephalin gene expression and binding of the transcription factor AP-2. Nucleic Acids Res 1990;18:3975-82.

37. Campanero MR, Armstrong MI, Flemington EK. CpG methylation as a mechanism for the regulation of E2F activity. Proc Natl Acad Sci USA 2000;97:6481-6.

38. Jones PL, Veenstra GJ, Wade PA, et al. Methylated DNA and MeCP2 recruit histone deacetylase to repress transcription. Nat Genet 1998; 19:187-91.

39. Nan X, Ng HH, Johnson CA, et al. Transcriptional repression by the methyl-CpG-binding protein $\mathrm{MeCP} 2$ involves a histone deacetylase complex. Nature 1998;393:386-9. 